\title{
In Memoriam Prof. Dr. İbrahim Mete Misırlığlu
}

(1972-2021)

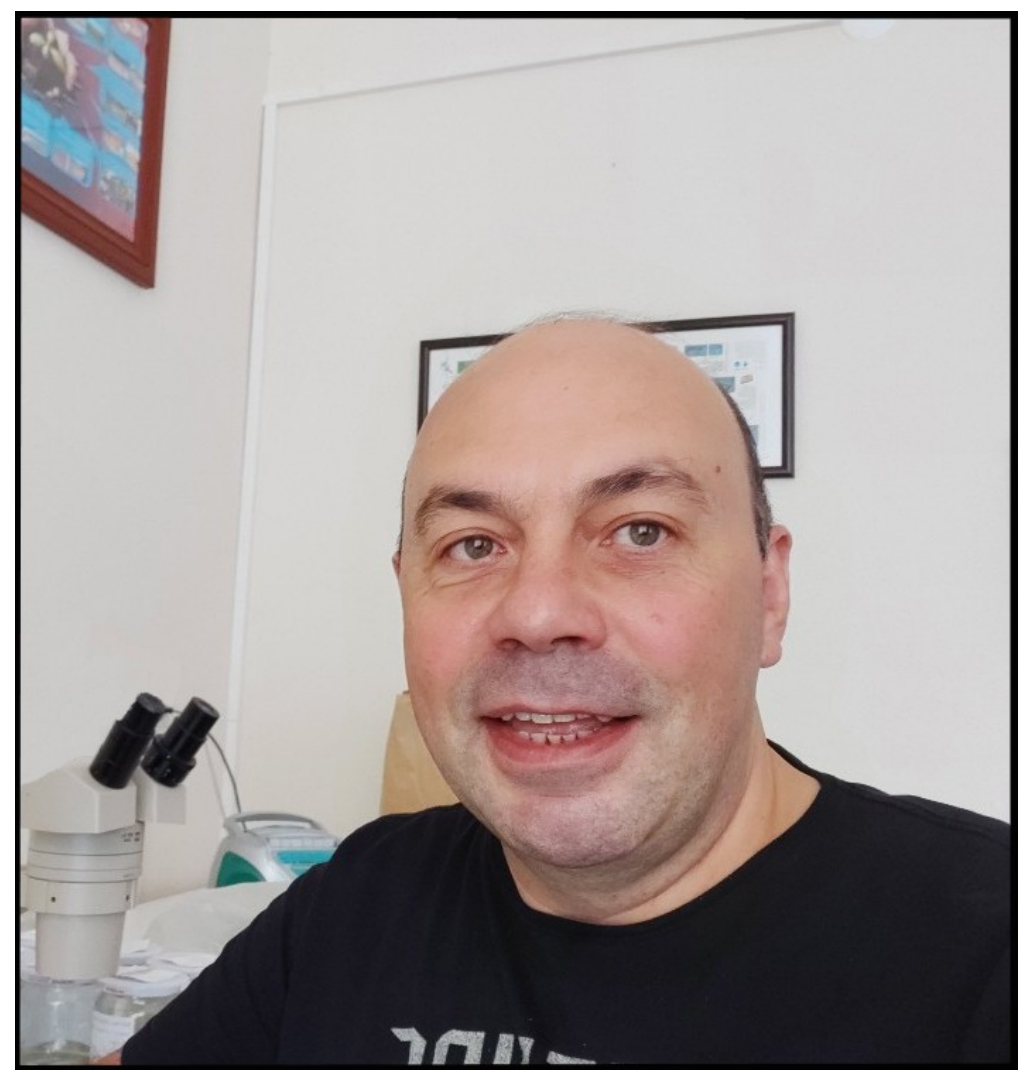

广brahim Mete Misırlığlu was a world renown earthworm taxonomist, and a valuable scientist both in Turkey and internationally. He passed away unexpectedly in 02 . May 2021 at age of 49 . He will be remembered as a leading soil zoologist in Turkey and the Middle East due to his outstanding contributions to oligochaete taxonomy, faunistics and biogeography and as a good person because of his charming personality and helpfulness.

İbrahim Mete Misırlığlu was born in 05. September 1972. He started his studies at the biology department of Anadolu University in 1989 and graduated from there in 1993. After graduating from the department of biology, he started his master's degree in the biology department of the Eskişehir Osmangazi University and graduated in 1995. He earned his Doctor of Philosophy (PhD) degree at the same university in 2001 and ever since he was working there, first as a research assistant at the Department of Biology, Science and Literature Faculty of Eskişehir Osman Gazi University. In 2013 he was appointed as an Associate Professor and in 2018 received the title of Full Professor.

He was working as a farabi coordinator between 2009-2021, and between 2013-2021 as the head of the biology department. The rapid rise in his academic career shows how talented a scientist he was. 
During his academic career, Prof. Misırlığlu has written 10 books, 1 chapter and some 90 articles, making great contributions to the knowledge on earthworms, especially in Turkey and the East Mediterranean. Besides his scientific activity he was a prolific popular science writer as well writing some 50 popular science articles on diverse topics like the Anatolian leopard or loss of Anatolian biodiversity.

He was full of plans, especially writing a book on earthworms for children and also a review paper on the world distribution of earthworm families and genera. Unfortunately, these plans

\section{Complete list of publications of Prof. Dr. İbrahim Mete Mısırlığlu}

\section{Articles published in SCI, SSCI or AHCI indexed journals}

CsuzDI, Cs., ZICSI, A. \& MisiRLioĞLU, I.M. (2006): Annotated checklist of the earthworm fauna of Turkey (Oligochaeta: Lumbricidae). Zootaxa, 1175: 1-29. doi: 10.11646/zootaxa.1175.1.1

Csuzdi, Cs., PAVlíčEK, T. \& MisirlioĞLU, I.M. (2007): Earthworms (Oligochaeta: Lumbricidae, Criodrilidae and Acanthodrilidae) of Hatay Province, Turkey, with description of three new lumbricids. Acta Zoologica Academiae Scientiarum Hungaricae, 53(4): 347-361.

MisiRLIOĞLU, I.M. (2008): A preliminary study of earthworms (Oligochaeta, Lumbricidae) from the city of Izmir, Turkey. Turkish Journal of Zoology, 32(4): 473-475.

MisIRLIOĞLU, I.M. (2008): Some earthworm records from Anatolia (Oligochaeta, Lumbricidae). Turkish Journal of Zoology, 32(4): 469-471.

MisIRLIOĞLU, I.M. (2010): Distribution of endemic earthworm species in Turkey (Oligochaeta: Lumbricidae). Zoology in the Middle East, 51(suppl. 2): 83-87. doi: $\underline{10.1080 / 09397140.2010 .10638461}$

PAVlíČEK, T., CSUZdi, Cs., MisirlioĞLU, I.M. \& VILENKIN, B. (2010): Faunistic similarity and endemism of earthworms in East Mediterranean region. Biodiversity and Conservation, 19(7): 1989-2001. doi: $10.1007 / \mathrm{s} 10531-010-9821-1$ remain to be accomplished by his students and colleagues around the world.

Certainly, Prof. Dr. İbrahim Mete Misirlıoğlu will sadly be missed by us, his students, and also his colleagues and friends both in Turkey and abroad.

\author{
İbrahim Tavuç \\ Department of Forest Engineering \\ Isparta University of Applied Sciences, \\ Isparta, Turkey.
}

Misirliö̆LU, I.M., Tsekova, R. \& Stojanović, M. (2016): On the presence of Lumbricus terrestris Linnaeus, 1758 (Oligochaeta, Lumbricidae) on the Balkan Peninsula: some aspects of ecology and distribution. Turkish Journal of Zoology, 40(3): 438-444. doi: 10.3906/zoo-1509-12

MisiRLIOĞLU, I.M. \& STOJANOVIĆ, M. (2017): Distribution and biogeographical significance of the endemic genera Spermophorodrilus Bouché, 1975 and Healyella Omodeo \& Rota, 1989 (Oligochaeta: Lumbricidae): a Review. Acta Zoologica Bulgarica, 69(1): 3-8.

VALChOVSKI, H. \& MisiRLIOĞLU, I.M. (2017): Murchieona minuscula (Rosa, 1906): first finding from Bulgaria with earthworm diversity and zoogeography of Yildiz (Strandja) Mountain in Turkey and Bulgaria. Turkish Journal of Zoology, 41(4): 731-736. doi: 10.3906/zoo-1605-46

MisIRLIOĞLU, I.M. (2018): Earthworms from Bursa, Uludag Mountain, with first record of Octolasion cyaneum (Savigny, 1826) from Turkey. Zootaxa, 4394(1): 141-143. doi: 10.11646/zootaxa.4394.1.10

VAlchovski, H., MisirlioğLU, I.M. \& StOJANOVIĆ, M. (2018): New earthworm records (Clitellata: Lumbricidae) from Bulgaria since 2012. Zootaxa, 4496(1): 173-174. doi: 10.11646/zootaxa.4496.1.12

MisirlioĞLU I.M. \& StOJAnOviĆ, M. (2018): Distribution of non-lumbricid earthworms (Clitellata: Acanthodrilidae, Criodrilidae, Megascolecidae and Ocnerodrilidae) on the Balkans and Anatolia with first record of Amynthas morrisi (Beddard, 1892) from Turkey. Zootaxa, 4496(1): 197-205. doi: $\underline{10.11646 / \text { zootaxa.4496.1.15 }}$ 
MisirlioĞLU, I.M., StojanOVIC M. \& TSEKOVA, R. (2018): Species richness of the earthworm fauna (Clitellata: Acanthodrilidae, Lumbricidae) of the Marmara Region in Turkey: Zoogeographical overview. North-Western Journal of Zoology, 14(2): 259-264.

\section{Scientific articles published in other journals}

MisIRLIOĞLU, I.M. (2002): The earthworms (Oligochaeta, Lumbricidae) of Eskişehir City, Turkey. Megadrilogica, 9(9): 17-20.

MisiRlioĞLU, M. (2003): Some Chilopoda (Myriapoda) records from the City of Eskişehir. Turkish Journal of Zoology, 27: 39-41.

MISIRLIOĞLU, I.M. (2004): Earthworm records from different parts of Anatolia. Megadrilogica, 10(1): $1-4$.

MisIRLIOĞLU, I.M. (2009): Current checklist of terrestrial Turkish earthworms (Oligochaeta). Megadrilogica, 13(3): 21-24.

DEMIROĞLU, G. \& MisirlioĞLU, I.M. (2010): Eskişehir ve Civarı Hirudinea Faunası Üzerine Bir Ön Araştırma [Preliminary study on the Hirudinea fauna of Eskişehir and its sourroundings]. Istanbul Commerce University Journal of Science, 17: 1925 .

TAŞTAN, N.S. \& MISIRLIOĞLU, I.M. (2010): Eskişehir İli Şehir Merkezi Karasal Gastropoda Türlerinin Belirlenmesi [Identification of terrestrial Gastropoda species in Eskișehir city center]. Istanbul Commerce University Journal of Science, 17: 2734.

SEMIZ, A. \& MisirLIOĞLU, I.M. (2010): Bozdağ ve Sündiken Dağları Karasal Gastropoda Türlerinin Belirlenmesi [Identification of terrestrial Gastropoda species in Bozdağ and Sündiken Mountains]. Istanbul Commerce University Journal of Science, 17: $35-44$

MisirlioĞLU, M. \& TAŞTAN, S., (2011): A preliminary study on earthworms (Oligochaeta, Lumbricidae) of Bursa city. Dumlupinar University Journal of Science, 25: 19-21.

Türkmen, C., Temel, E., Çatal, G., Sinecen, M. \& MISIRLIOĞLU, I.M. (2013): Bazı Atık ve Toprak Düzenleyicilerin Toprakta Solucan Davranışlarına Etkisi [Effect of Some Waste and Soil Regulators on Worm Behavior in Soil]. COMU Journal of Agriculture Faculty, 1: 79-86.

MisiRlioĞLU, M. \& SzEderJesi, T. (2015): Contributions to the earthworm fauna of Turkey. Megadrilogica, 18(6): 99-102.

MisiRLIOĞLU, I.M. \& VALCHOVSKI, H. (2016): Distribution ecology and zoogeography of the genus Fitzingeria (Oligochaeta, Lumbricidae). Megadrilogica, 20(8): 137-142.

MisiRLIOĞLU, I.M. \& VALCHOVSKI, H. (2017): Contributions to earthworm (Clitellata Annelida) fauna of Turkish Thrace. Sakarya University Journal of Science, 21(3): 385-388. doi: 10.16984 /saufenbilder.298983

VAlChOVsKi, H. \& MIsIRLIOĞLU, I.M. (2017): Review of earthworm (Clitellata: Lumbricidae, Criodrilidae, Acanthodrilidae) biodiversity of Thrace in Bulgaria, Turkey and Greece. Sakarya University Journal of Science, 21(6): 1325-1330. doi: 10.16984/saufenbilder.306021

MISIRLIOĞLU, I.M. (2017): Diversity of earthworm (Clitellata, Annelida) species in the Asian and European part of Turkey. KSU Journal of Agriculture and Nature, 20(2): 115-119. doi: $\underline{10.18016 / \text { ksujns.71383 }}$

MisiRlioĞLU, I.M. \& VALCHOVSKI, H. (2017): Preliminary research on the earthworm (Clitellata; Lumbricidae) fauna of Eskisehir Besik Deresi and Eskisehir-Mihaliccik Gurleyik Village. $K S U$ Journal of Agriculture and Nature, 20(4): 398-401. doi: $\underline{10.18016 / \mathrm{ksudobil} .286036}$

SzEdERJESI, T. \& MisirlioĞLU, I.M. (2017): New earthworm records from Turkey (Clitellata: Lumbricidae, Megascolecidae). Opuscula Zoologica (Budapest), 48: 55-60. doi: 10.18348/opzool.2017.1.55

MisirLIOĞLU, I.M., ŞEN, O \&, TEMEL, V. (2018): Earthworm (Clitellata, Annelida) records from Eskişehir, Sakarya and Düzce Provinces, Turkey. KSU Journal of Agriculture and Nature, 21(3): 424-427. doi: 10.18016/ksudobil.346295

MisiRlioĞLU, I.M., VAlChOVSKi, H. \& StojanOVić, M. (2018): Review of the earthworm biodiversity of Turkey and its neighbouring countries (Clitellata, Megadrili). Opuscula Zoologica Budapest, 49(2): 141-149. doi: 10.18348/opzool.2018.2.141

REYNOLDS, J.W. \& MisirlioĞLU, I.M. (2018): Preliminary key to Turkish megadriles (Annelida, 
Clitellata, Oligochaeta), based on external characters, insofar as possible. Megadrilogica, 23(11): 141-160.

MisirLIOĞLU, I.M., VAlChOVSKI, H. \& REYNOLDS, J.W. (2019): Updated list of earthworm species from Turkey (Clitellata, Megadrili). Megadrilogica, 24(8): 99-106.

MISIRLIOĞLU, I.M. (2019): On the distribution of Helodrilus patriarchalis (Rosa, 1893) (Clitellata, Megadrili) in Turkey, with new record from Eskişehir Province, Central Anatolia. Megadrilogica, 25(1): 18-20.

MisIRLIOĞLU, I.M. \& REYNOLDS, J.W. (2019): On the distribution of the genus Octodrilus (Clitellata, Megadrili) in Turkey. Megadrilogica, 25(2): 33-37.

MisirlioĞLu, I.M., TseKova, R., \& VALChOVSKI, H. (2019): Distribution of Atlanto-Mediterranean and Balkan-Anatolian earthworm species (Clitellata, Megadrili) in Turkey. Megadrilogica, 25(3): 46-51.

MisiRLIOĞLU, I.M. \& VALCHOVSKI, H. (2019): Earthworm (Clitellata Megadrili) records from Eskişehir Province. Afyon Kocatepe University Journal of Science and Engineering, 19: 533-535. doi: 10.35414/akufemubid.514127

MISIRLIOĞLU, I.M. \& VALCHOVSKI, H. (2019): Earthworm (Clitellata Megadrili) records from Adana Province. Sakarya University Journal of Science, 23(6): 1106-1109. doi: 10.16984/saufenbilder.431200

MISIRLIOĞLU, I.M. (2019): Eisenia andrei (Bouché, 1972) (Clitellata: Megadrili) Türünün Türkiye'den Doğal Habitatta İlk Kaydı [First record of Eisenia andrei (Bouché, 1972) (Clitellata: Megadrili) from natural habitat in Turkey]. Afyon Kocatepe University Journal of Science and Engineering, 19: 264-266. doi: 10.35414/akufemubid.496669

MisirlioĞLu, I.M. \& StOJANović, M. (2020): Dendrobaena attemsi (Michaelsen, 1902) (Clitellata, Megadrili) on the Balkan Peninsula and Anatolia: Distribution and biogeographical significance. Opuscula Zoologica (Budapest), 51(2): 123-131. doi: $\underline{10.18348 / \text { opzool.2020.2.123 }}$

MisiRLIOĞLU, I.M., STOJANOVIĆ, M. \& REYNOLdS, J.W. (2020): Distribution of Aporrectodea handlirschi mahnerti (Zicsi, 1973) (Clitellata, Megadrili) in Turkey. Megadrilogica, 25(11): 127-130.

MisiRLIOĞLU, I.M. \& REYNOLDS, J.W. (2021): Distribution of Dendrobaena pentheri (Rosa, 1905) (Clitellata, Megadrili) in Turkey and the region. Megadrilogica, 26(4): 61-64.

\section{Books \& Book Chapters}

MisiRlioĞLU, I.M. (2011): Omurgasız Hayvanlar Laboratuvar Kılavuzu. [Invertebrate animals; Laboratory manual] Nobel Publisher, Ankara.

MISIRLIOĞLU, I.M. (2011): Toprak solucanlarl, Biyolojileri, Ekolojileri ve Türkiye Türleri. [Earthworms; their biology, ecology and the Turkish species] Nobel Publisher, Ankara.

MisIRLIOĞLU, I.M. (2014): Toprak Faunast. [Soil fauna] Nobel Publisher, Ankara.

MISIRLIOĞLU, I.M. (2015): Omurgasiz Hayvanlar Laboratuvar Kilavuzu Gözden Geçirilmiş 2. Baski. [Invertebrate animals; Laboratory guide manual. Revised 2nd edition] Nobel Publisher, Ankara.

MISIRLIOĞLU, I.M. (2017). Toprak Solucanlarl, Biyolojileri, Ekolojileri, Zirai Yönleri, Türkiye Türleri ve Türlerin Taksonomik Özellikleri. Genişletilmiş ve Gözden Geçirilmiş 2. Basim [Earthworms, their biology, ecology, Agricultural Aspects; Turkish species and their taxonomic characteristics. Extended and revised 2nd edition] Nobel Publisher, Ankara.

MisIRLIOĞLU, I.M. (2018). Türkiye Faunası ÜzerineDers Notlarl [Lecture notes on the fauna of Turkey]. Eskişehir Osmangazi Üniversitesi Yayinevi, Eskişehir.

MISIRLIOĞLU, I.M. (2018): Omurgasiz Hayvanlar Laboratuvar Kllavuzu [Invertebrates laboratory guide]. Eskişehir Osmangazi Üniversitesi Yayinevi, Eskişehir.

MISIRLIOĞLU, I.M. (2018): Türkiye Toprak Solucanı Atlası [Earthworm atlas of Turkey]. Eskişehir Osmangazi Üniversitesi Yayınevi, Eskişehir.

MISIRLIOĞLU, I.M. (2020): Karasal Ekosistemler [Terrestrial ecosystems]. Eskişehir Osmangazi Üniversitesi Yayınevi, Eskişehir.

MisiRLIOĞLU, I.M. (2020): Sistematik ve Taksonomi. [Systematics and taxonomy]. Eskişehir Osmangazi Üniversitesi Yayınevi, Eskişehir.

\section{Publications in Congress / Symposium Proceedings}

MisiRlioĞLU, I.M., PAVlí̌čEK, T. \& Csuzdi, Cs. (2007) Earthworm Biodiversity in Turkey: An Overview. 3rd International Oligochaeta Taxonomy Meeting, Platres, Cyprus, 02-06 April 2007. 
ÖZDEN, H., ŞentürK, H., GÜVen, G., KABAy, Ş., ÖZ, S., MisirlioĞLU, I.M., BURUKOĞLU DöNMEZ, D., ÖZBAYer, C., YILdiz, F., KaYgisiz, B., et al. (2012): The effects of Lumbricus extract on the antioxidant system and histological structure of nephrectomized rats in a ischemia-reperfusion model. Fourth B \& H Symposium "Morphology in science and practice" with international participation, Sarajevo, Bosnia and Herzegovina 27-30 September 2012.

Pavlíček, T., Hadid, Y., Cohen, T., Glastetter, M., Snir, S., MisirlioĞLU, I.M., Pearlson, O., YADAV, S., CSUZDI, CS. \& KRAL, P. (2013): Opening Pandora's Box" II. Segmentation and evolution of hermaphroditic annelids. $6^{\text {th }}$ International Oligochaeta Taxonomy Meeting, Palmeira de Faro, Portugal, 22-25 April 2013.

CANBazk, S., ÖZ, S., ÖZden, H., BURUKoĞLU DöNMEZ, D., Kus, G., ÜsTÜNER, M.C., ŞENTÜRK, H., MisiRLIOĞLU, I.M., YILdIZ, F. \& AYDEMIR, D. (2014): The evaluation of the protective effect of Lumbricus extract for brain and the effect on antioxidant system in unilateral common carotid artery ischemia/reperfusion model. 16 ${ }^{\text {th }}$ National Congress of Anatomy, Malatya, Turkey, 11-14 September 2014.

YILMAZ, S. \& MisIRLIOĞLU, I.M. (2014): Soğan Yetiştiriciliğinde Solucan Gübresinin Etkilerinin Incelenmesi [Investigation the effects of vermicompost on onion growing]. 22. Ulusal Biyoloji Kongresi, Eskişehir, Turkey, 23-27 June 2014

MisirLIOĞLU, I.M. \& Temel, V. (2016): Türkiye Toprak Solucanı Faunasina (Oligochaeta, Annelida) Genel Bakiş ve Güncel Tür Listesi [Earthworm fauna of Turkey; overview and current species list]. 23. Ulusal Biyoloji Kongresi, Gaziantep, Turkey, 05-09 September 2016.

MisiRLIOĞLU, I.M. \& VALCHOVSKI, H. (2016): Species richness of the Dendrobaena (Oligochaeta Lumbricidae) genus in Turkey and Bulgaria. Seminar of Ecology with International Participation, Sofia, Bulgaria, 21-22 April 2016

MisiRLIOĞLU, I.M. \& ŞEN, O. (2016): Anadolu ve Trakya'nın Toprak Solucanı (Oligochaeta, Annelida) Türleri [Earthworm species of Anatolia and Thrace]. Ulusal Uygulamalı Biyolojik Bilimler Kongresi. Konya, Turkey, 26-29 December 2016.

MisirLIOĞLU, I.M. \& ŞEN, O. (2016): Determination results of some earthworms Samples collected from
Turkish Thrace. International Conference on Biological Sciences (ICBS) Konya, Turkey, 21-23 October 2016

MisiRlioĞLU, I.M., ARDiÇ, M., Metin, R. \& ÖZÇELEBI, Ö. (2016): Toprak Solucanlarının Toprağın Organik Madde Miktarı, pH Değeri Üzerine Etkileri ve Vermikompost Oluşumundaki Rollerinin İncelenmesi [Investigation the effects of earthworms on soil organic matter content and $\mathrm{pH}$, and their role in vermicompost formation.] 3. Ulusal Çevre Kongresi, Muğla, Turkey, 24-28 September 2016.

MisiRlioĞLu, I.M., TSEKova, R. \& StoJanović, M. (2016): Distribution of the genus Cernosvitovia (Oligochaeta, Lumbricidae) on the Balkan Peninsula. $5^{\text {th }}$ Congress of Ecologists of the Republic of Macedonia with International Participation. Ohrid, Macedonia, 19-22 October 2016.

MisiRLIOĞLU, I.M., VAlchovski, H. \& TSEKova, R. (2016): Peregrine earthworms (Clitellata Lumbricidae) from Bulgaria and Turkey. Klimentovidni Conference, Sofia, Bulgaria, 17-18 November 2016.

ŞEN, O., AKÇINAR, O. \& MisiRlioĞLU, I.M. (2016): Solucan Gübresinin Havuç Bitkisi Gelişimine Olan Etkileri Üzerine Bazı Gözlemler [Observations on the effects of vermicompost on growth of carrot.] Ulusal Uygulamalı Biyolojik Bilimler Kongresi. Konya, Turkey, 26-29 December 2016.

Valchovski, H., MisirlioğLU, I.M. \& Tsekova, R. (2016): First record of Aporrectodea jassyensis Michaelsen, 1891 (Clitellata, Lumbricidae) from the Rila Mountain. Klimentovidni Conference, Sofia, Bulgaria, 17-18 November 2016.

MisiRLIOĞLU, I.M., ŞEN, O. \& TEMEL, V. (2017): Vermicompost and Vermiculture. International Symposium on Biodiversity and Edible Wild Species (BEWS 2017) Antalya, Turkey, 03-05 April 2017.

MisirLIOĞLU, I.M., ŞEN, O. \& TEMEL, V. (2017): Earthworm (Clitellata, Annelida) records from Eskişehir, Sakarya and Düzce Province. International Conference on Agriculture, Forest, Food Sciences and Technologies (ICAFOF, 2017). Cappadocia / Nevşehir, Turkey, 15-17 May 2017.

Şen, O., MisirlioĞLU, I.M. \& Temel, V. (2017): Vermikompost. II [Vermicompost II]. Ulusal Uygulamalı Biyolojik Bilimler Kongresi. Afyon, Turkey, 09-12 July 2017.

MisiRlioĞLU, I.M., AKÇINAR, O., VALChOVSKI, H., TEMEL,V., ŞEN, O. \& YALÇIN, E. (2018): Medical 
Aspects of Earthworms. IV. International Congress on Applied Biological Sciences, 03-05 May 2018.

MisiRlioĞLU, I.M., YALÇIN, E., VAlChOVSKI, H. \& ŞEN, O. (2018): Quantitative comparison of the species diversity of earthworms (Clitellata: Annelida) of Turkey with other countries. International Symposium of Ecology. Kastamonu, Turkey, 1923 June 2018.

TAVuÇ, I., MisirlioğLU I.M., KAPTANOĞLU, A.S. \& ÇELIK, N. (2018): A Preliminary investigation on the earthworm (Clitellata, Megadrili) distribution in different stands on Türkmen Mountain. International Conference on Science and Technology (ICONST 2018). Prizren, Serbia, 05-09 September 2018.

MisirlioĞLU, I.M., TSEKOVA, R. \& VALChOVSKI, H. (2019): Distribution of the Balkan-Anatolian earthworms (Clitellata, Megadrili) Dendrobaena cognettii (Michaelsen, 1903) and Eisenia kattoulasi Zicsi \& Michalis, 1981. Klimentovidni Conference, Sofia, Bulgaria, 08 November 2019.

\section{Popular science articles}

MisiRlioğLU, I.M. (2001): Doğanin Gönüllü Bahçivanları: Toprak Solucanlari [Earthworms; the nature's voluntary gardeners]. Bilim ve Teknik Dergisi, 408: 78-80.

MisIRLIOĞLU, I.M. (2001): Toprak Solucanları ve Ekolojik Önemleri [Earthworms and their ecological significance]. Popüler Bilim Dergisi, 96: 21-23.

MisiRLIOĞLU, I.M. (2002): Anadolu'nun Büyük Kedileri [Big cats of Anatolia]. Popüler Bilim Dergisi, 99: $37-38$.

MisiRLIOĞLU, I.M. (2002): Karacalar Capreolus capreolus [The roe deer]. Popüler Bilim Dergisi, 103: 39-40.

MisIRLIOĞLU, I.M. (2002): Mavi Denizlerin Sevimli Memelileri Yunuslar [Cute Mammals of the Blue Seas: The Dolphins]. Bilim ve Teknik Dergisi, 420: 93.

MISIRLIOĞLU, I.M. (2002): Ormanin Vahşi Kedileri Vaşaklar [Lynxes; wild cats of the forests]. Bilim ve Teknik Dergisi, 411: 86-87.

MisIRLIOĞLU, I.M. (2003). Zehirli Hayvanlar [Poisonous animals]. Bilim ve Teknik Dergisi, 428: 91.
MisIRLIOĞLU, I.M. (2003): Ç1yanlar [Centipedes]. Popüler Bilim Dergisi, 110: 52-53.

MisIRLIOĞLU, I.M. (2003): Ulu Geyikleri Yaşatalım [Let's keep the great deer alive]. Popüler Bilim Dergisi, 116: 39.

MisiRlioĞLU, I.M. (2003): Bir Vahşi Anadolu Kedisi Karakulak [Caracal caracal; an Anatolian wildcat]. Popüler Bilim Dergisi, 118: 12-14.

MisiRLIOĞLU, I.M. (2003): Solucan Deyip Geçmeyin [Do not say worm]. Buğday Dergisi, 19: 40.

MISIRLIOĞLU, I.M. (2004): Kaybolan Biyolojik Zenginliklerimiz Soyu Tükenen Memeliler [Our lost biodiversity; extinct mammals]. Bilim ve Teknik Dergisi, 441: 88-91

MISIRLIOĞLU, I.M. (2004). Ülkemizin Efsane Kedisi Anadolu Leoparı [Anatolian leopard; the legendary cat of our country]. Bilim ve Teknik Dergisi, 456: 32 .

MisIRLIOĞLU, I.M. (2004). Ulu Geyikler [Great dear]. Bilim ve Çocuk Dergisi, 82: 32-33.

MiSIRLIOĞLU, I.M. (2004): Toprak Solucanlarının Yararı Nedir? [What is the benefit of earthworms?] Hürriyet Bilim Dergisi, 135: 19.

MisiRLIOĞLU, I.M. (2005): Soyu Tükenen Türlerin Korunması İçin Ne yapılıyor [What is being done to protect endangered species]. Cumhuriyet BilimTeknik, 960: 18-19.

MisiRLIOĞLU, I.M. (2006): Doğadaki Besin Ağı ve Soyu Tükenen Canlılar [Natural foodweb and endangered species]. Popüler Bilim Dergisi, 148: $52-54$.

MISIRLIOĞLU, I.M (2007): Toprak Hayvanları [Soil animals]. Popüler Bilim Dergisi, 165: 18-23.

MISIRLIOĞLU, I.M. (2007): Toprağın Devleri [Giants of the soil]. Bilim ve Teknik Dergisi, 470: 25.

MisIRLIOĞLU, I.M. (2007): Toprak Altında Yeni Türler [New subterranean species]. National Geographic Türkiye, 76: 26.

MiSIRLIOĞLU, I.M. (2007): Toprak Solucanları [Earthworms]. TEMA, xx:zz.

MisiRLIOĞLU, I.M. (2010): Toprağın Sessiz İşçileri Solucanlar [Worms, the silent workers]. Ekoloji Magazin Dergisi, 25: 26-29. 
MisiRLIOĞLU, I.M. (2010): Toprak Sihirbazı Hastaliklara Derman Oluyor [Earth wizard cures diseases]. NTV Bilim Dergisi, 13: 56.

MisiRlioĞLU, I.M. (2010): Türkiye Faunası Köşe Yazısı [Fauna of Turkey column]. Eskişehir Istikbal Gazetesi.

MisiRlioĞLU, I.M. (2010): Türlerin Yok Oluşu I [Extinction of species I]. Eskişehir Istikbal Gazetesi.

MisiRLIOĞLU, I.M. \& ŞAHIN, Y. (2011): Toprak solucanlarının Toprağa ve Tarıma Etkileri [Effect of earthworms on soil and agriculture]. Hasad Dergisi, 313: 78-81.

MisiRLIOĞLU, I.M. (2011). Türlerin Yok Oluşu II KöşeYazisi [Extinction of Species Column II]. Eskişehir Istikbal Gazetesi.

MisiRlioĞLU, I.M. (2011): Anadolu Leoparı Köşe Yazısı [The Anatolian Leopard Column]. Eskişehir Istikbal Gazetesi.

MisIRLIOĞLU, I.M. (2011): Anadolu'da Kaplan [Tiger in Anatolia]. Esogü Haber Dergisi, 125: 7.

MisiRLIOĞLU, I.M. (2011): Anadolu ve Yok Olan Zenginliklerimiz [Anatolia and our lost wealth]. Esogü haber Dergisi, 129: 9.

MisIRLIOĞLU, I.M. (2011): Kaplanın Öyküsü Köşe Yazısı [The Tiger's tale]. Eskişehir Istikbal Gazetesi.

MisiRLIOĞLU, I.M. (2011): Mutfakta Çalışan Solucanlar [Worms working in the kitchen]. National Geographic Türkiye, 122: 68.

MisIRLIOĞLU, I.M. (2011): Toprağin Sihirbazları Toprak solucanlar1 [Wizzards of the earthworms]. Bilim ve Teknik Dergisi, 529: 70-72.

MisIRLIOĞLU, I.M. (2011): Toprak solucanları Köşe Yazısı [Earthworms column]. Eskişehir Istikbal Gazetesi.

MisIRLIOĞLU, I.M. (2012): Bir zamanlar Eskişehir de [Once in Eskişehir]. Eskiyeni-Eskişehir Valiliği Dergisi, 42: 58-59.

MisiRLIOĞLU, I.M. (2012): Türkiye Faunası [Fauna of Turkey]. Esogü haber Dergisi, 137: 9.
MisIRLIOĞLU, I.M. (2014): Toprak solucanları şifa kaynağımı [Earthworms are our source of healing ?]. National Geographic Türkiye, 160: 28.

MisIRLIOĞLU, I.M. (2016): Solucan Gübresi nedir [What is vermicompost ?] Bilim ve Teknik Dergisi, 586: 46 .

MisIRLIOĞLU,I.M. (2016): Toprak solucanlar1; Ekolojik Sürpriz [Earthworms; ecological surprise]. Atlas Dergisi, 279: 26.

MisIRLIOĞLU, I.M. (2017): Solucan Gübresi Yayginlaşıyor [The vermicompost is sperading]. Atlas Dergisi, xx: zz.

MisiRLIOĞLU, I.M. (2018): Solucan deyip geçmeyin 2 [Do not say worm 2]. Herkese Bilim Teknoloji Dergisi, 103: 7 .

MisIRLIOĞLU, I.M. (2018): Toprak Faunası [Soil fauna]. Popüler Bilim Dergisi, 258: 44-45.

MisIRLIOĞLU, I.M. (2018): Toprak solucanları Şifa Kaynağı Olabilir mi? [Can earthworms be a source of healing ?] Magma Dergisi, 32: 18.

MISIRLIOĞLU, I.M. (2018): Toprak solucanları Marsa Giderse [If earthworms go to Mars]. Magma Dergisi, 35: 16.

MisiRLIOĞLU, I.M. (2018): Toprak Altındaki Müttefikler [Allies underground]. Magma Dergisi, 43: 23.

MisIRLIOĞLU, I.M. (2018): Toprak solucanları: Doğanin emekçileri [Earthworms: workers of the Nature]. Atlas Dergisi, 304: 12.

MiSIRLIOĞLU, I.M. (2019): Dev Toprak solucanları Yer altının Devleri [Giant earthworms - giants of the underground]. Atlas Dergisi, 317: 22.

MisiRLIOĞLU, I.M. (2019): Türkiye Toprak solucanı Tür Sayısı 87'ye yükseldi [Turkey's earthworm species number increased to 87]. Herkese Bilim Teknoloji Dergisi, 167: 16.

MisiRLIOĞLU,I.M. (2019): Zoolojik Servetimiz - Türkiye Faunas1. [Our zoological wealth - Turkish fauna]. Bilim ve Teknik Dergisi, 623: 72-83. 